\section{Reclamos por atención en salud. Experiencia en un centro privado}

\author{
RICARDO ESPINOZA GONZÁLEZ1 ${ }^{1}$, XIMENA SALCEDO ${ }^{1, \mathrm{a}}$, \\ SERGIO SAN MARTÍN RAMÍREZ ${ }^{1}$
}

\section{Analysis of healthcare claims received at an audit unit of a clinical hospital}

Background: The resolution of claims at healthcare institutions is an important issue for administrators. Aim: To analyze the causes of healthcare-related claims in a private, clinical health center. Material and Methods: All claims processed at the audit unit of a clinical hospital between 2016 and 2019 were analyzed. Results: In the study period, 602 complaints were recorded, representing a rate of one claim for every 1,000 services provided. The two main causes of complaints were sub-standard interaction with the doctor such as communication or lack of empathy, corresponding to $21 \%$ of cases, and complaints related to care costs, quotes, and insurance coverage (21\%). The highest number of complaints originated in emergency care, but the highest rate by number of healthcare services provided occurred in hospitalization in the Mental Health and the Adult Critical Care units. Roughly, 75\% of the complaints were settled with a phone call to the patient, which allowed to explain what happened and to apologize accordingly. In $21 \%$ of the cases, one or more meetings were held with the patient, a family member, or his/her legal representative to settle an agreement. In 3.5\% of cases a mediation process through an independent third party was required. Only $1 \%$ of the claims resulted in a legal action. Conclusions: The importance of cultivating an adequate physician-patient relationship for the prevention of complaints and eventual litigations associated with the provision of healthcare services, is emphasized. Quality control policies and processes within healthcare organizations should be strengthened.

(Rev Med Chile 2021; 149: 1311-1316)

Key words: Empathy; Ethics; Malpractice; Negotiating; Physician-patient relationship.

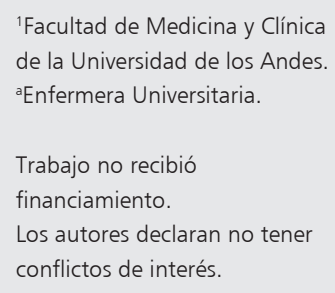

$\mathrm{E}$ 1 año 2012 entró en vigencia en Chile la Ley $n^{\circ} 20.584$ que regula los derechos y deberes de las personas en relación a su atención en salud $^{1,2}$. De esta forma, y bajo protección legal, el Estado quiso que se resguardaran aspectos de la seguridad del paciente y la calidad de las atenciones. Esto último se refiere a que, independiente del prestador, sea público o privado, a cada persona se asegure un trato digno, una atención amable, y prestaciones otorgadas según protocolos establecidos, entre otros derechos. Al mismo tiempo, el
Título IV de la misma Ley dejó establecido que la persona puede reclamar ante el establecimiento donde recibió la atención, en la medida que sienta vulnerado sus derechos. Es mandatorio responder el reclamo, por escrito, en un plazo máximo de 15 días hábiles.

Por tanto hoy, en cada centro de atención en salud, se ha estructurado orgánicamente, una unidad o departamento que, entre otras funciones, recoge, analiza y responde los reclamos, unidad usualmente conocida como Auditoría Médica ${ }^{3}$. 
La primera instancia de resolución de un reclamo recae, por tanto, en la Auditoría del propio centro. De no existir acuerdo, se contempla que la persona realice una presentación ante un organismo superior. En el caso de un reclamo originado en el ámbito de un prestador privado, se puede realizar un planteamiento ante la Superintendencia de Salud; ésta puede acoger el reclamo y ofrecer la posibilidad de una mediación para resolverlo. Cuando el prestador es público, la mediación se realiza por medio del Consejo de Defensa del Estado, de modo gratuito. En cualquiera de los casos, el objetivo es intentar que se llegue a la judicialización. La mediación, como herramienta de resolución prejudicial de conflictos en salud quedó establecida por la Ley 19.966 de Régimen de Garantías en Salud, que data del año $2004^{4}$. Esta modalidad, que intenta recuperar la relación médico-paciente, ha visto un significativo incremento en su demanda desde su implementación, al menos en el ámbito público por información proveniente del Consejo de Defensa del Estado ${ }^{5}$.

La tasa de reclamos por atenciones efectuadas, su naturaleza y la forma de resolverlos habitualmente queda en los registros internos de los centros. En Chile, de experiencia de prestadores privados, sólo conocemos la publicada por Moore et $a^{6}$ en el Hospital Clínico de la Pontificia Universidad Católica de Chile, el año 2011. El objetivo del presente trabajo es analizar las causas y manejo de los reclamos en un centro privado de salud, también universitario, que presentaron pacientes o sus familiares, de acuerdo al derecho de las personas, consagrado por la referida Ley 20.584. Estimamos que el conocimiento de esta información es de gran utilidad para el propio centro donde se han generado los reclamos, como fuente de mejoría de la atención que, incluso, puede ser extrapolada a otros centros de salud.

\section{Material y Método}

De la totalidad de los reclamos atendidos por la Unidad de Auditoría Médica del Hospital Clínico de la Universidad de los Andes, en un período de cuatro años (2016-2019) se efectuó un análisis retrospectivo y estudio descriptivo de éstos. Toda la información relativa al reclamo fue manejada respetando la confidencialidad de la información, como la intimidad y dignidad de las personas. (Declaración de Helsinski, de la Asociación Médica
Mundial, 2013). La información clínica contenida en nuestro Registro Clínico Electrónico y la materia de cada reclamo son transmitidas por vía electrónica, pudiendo acceder a ellas sólo aquellas personas con el privilegio explícito otorgado por la Dirección del Establecimiento. Desde la Unidad de Control de Gestión se obtuvo el número total de prestaciones ambulatorias y el número de hospitalizaciones por servicio clínico, que conforman el universo de atenciones de donde se originaron los reclamos. De acuerdo a nuestra organización, los reclamos son recibidos por la oficina de Atención al Paciente y desde ahí derivados a la Unidad de Auditoría Médica, para su resolución. Esta Unidad está conformada por los autores del presente trabajo. Para este análisis se incluyeron los reclamos generados tanto en atención abierta como en el transcurso de una hospitalización. Se analizó el servicio clínico donde se generó el reclamo, la causa que lo motivó, la forma de resolución y el tiempo empleado para dar una respuesta.

No existe una clasificación universal sobre las causas que originan un reclamo; las diferentes publicaciones emplean distintas categorías e identifican diversos incidentes como generadores de éstos. Nuestra Unidad creó una clasificación, que es la utilizada en este análisis. Algunos reclamos implican más de un motivo; para el análisis, se consideró sólo el incidente más relevante, de acuerdo a la presentación escrita del reclamo y el criterio consensuado de los miembros integrantes de la Unidad de Auditoría. La categoría "expectativas" incluye una variedad de situaciones, como la disconformidad con el resultado de un tratamiento, las más de las veces posterior a cirugía plástica estética; a la oportunidad de efectuar algún examen; el retraso en la realización de un procedimiento, entre otras.

Para la resolución del reclamo, y de acuerdo a la complejidad de éste, aspecto también consensuado por los miembros de la Unidad, se recurrió a una llamada telefónica al paciente o familiar, o a la invitación para tener una reunión presencial. Por tanto, para el análisis de la resolución del conflicto, se clasificaron los reclamos entre aquellos en los que fue suficiente una llamada telefónica para atenderlo, (tipo A); los que requirieron de una o más entrevistas con el paciente o familiar, (tipo B); como reclamos tipo $\mathrm{C}$ se clasificaron aquellos que derivaron a una mediación y tipo $\mathrm{D}$, los que desembocaron en la apertura de un juicio civil. 


\section{Resultados}

En el período señalado la Unidad de Auditoría atendió 602 reclamos. Sobre el total de prestaciones, suma de consultas ambulatorias y hospitalizaciones, la frecuencia de reclamos fluctuó entre 0,07\%, cifra observada en el año 2016, y 0,12\% de todas las prestaciones otorgadas por el Centro, en el año 2019. Si bien el número absoluto de reclamos fue aumentado en relación al número de atenciones, en términos prácticos, se ha presentado de forma estable, cada año, un reclamo por cada 1.000 atenciones (Tabla 1).

Si observamos el número de reclamos por Servicio, es en Urgencia y en Centro Médico donde se genera la mayor cantidad de ellos; sin embargo, la tasa por número total de atenciones otorgadas por estos Servicios es de 0,14 y $0,02 \%$ respectivamente (Tabla 2). Las mayores tasas de reclamos se observan en hospitalización de Salud Mental (1,76\%) y en la Unidad de Paciente Crítico de Adulto (UPCA) con una tasa de 1,47\%.
$\mathrm{Al}$ analizar el total de reclamos, observamos que $22,6 \%$ se generó en Urgencia, seguida ésta del Centro Médico Ambulatorio con 17,6\% de los casos; en tercer lugar se ubicó el Pabellón Quirúrgico. Los servicios de Salud Mental y la UPCA pesan relativamente menos en el total de los reclamos (Tabla 3 ).

Las causas de los reclamos se observan en la Tabla 4. Fue la falla en la relación con el médico la más frecuente de ellas (21,4\% del total), seguida por los reclamos por cobros, presupuesto o coberturas de seguros. Observamos infrecuentemente, reclamos por errores asociados a medicamentos y por complicaciones de un tratamiento médico o quirúrgico. Reclamos por falla o retardo en el tratamiento o falla o retardo en el diagnóstico representan $6,1 \%$ y $9,5 \%$ del total de los reclamos, respectivamente.

Como se observa en la Tabla 5, 74,7\% de los reclamos se resolvió con una llamada telefónica dando las explicaciones de lo sucedido y ofreciendo las disculpas correspondientes. En 20,8\%

Tabla 1. Reclamos por año

\begin{tabular}{|cccc|}
\hline Año & n total prestaciones & n reclamos & Frecuencia \% \\
\hline 2016 & 92.076 & 66 & 0,07 \\
2017 & 127.205 & 144 & 0,11 \\
2018 & 169.927 & 150 & 0,09 \\
2019 & 196.444 & 242 & 0,12 \\
\hline
\end{tabular}

Tabla 2. Reclamos por Servicio Clínico

\begin{tabular}{|lccc|}
\hline Servicio & $\begin{array}{c}\text { n prestaciones por } \\
\text { Servicio (2016-2019) }\end{array}$ & $\begin{array}{c}\text { n reclamos por } \\
\text { Servicio (2016-2019) }\end{array}$ & $\begin{array}{c}\text { Frecuencia de reclamos } \\
\text { por Servicio \% }\end{array}$ \\
\hline Urgencia & 99.666 & 136 & 0,14 \\
\hline Centro médico & 499.203 & 106 & 0,20 \\
\hline Pabellón & 11.678 & 83 & 0,71 \\
Hospitalización médico-quirúrgica & 14.430 & 71 & 0,49 \\
\hline Imágenes & 194.908 & 36 & 0,02 \\
\hline Hospitalización maternidad & 4.372 & 32 & 0,73 \\
\hline Endoscopía & 9.533 & 24 & 0,25 \\
\hline Pediatría & 2.066 & 20 & 0,97 \\
\hline Salud mental & 1.080 & 19 & 1,76 \\
UPCA * & 1.088 & 16 & 1,47 \\
\hline
\end{tabular}

* Unidad de Paciente Crítico Adulto. 
Tabla 3. Participación de Servicios Clínicos en el total de reclamos

\begin{tabular}{|lc|}
\hline Servicio & $\begin{array}{c}\text { \% sobre el total } \\
\text { de reclamos }\end{array}$ \\
\hline Urgencia & 22,6 \\
\hline Centro médico & 17,6 \\
\hline Pabellón & 13,8 \\
\hline Hospitalización médico quirúrgica & 11,8 \\
\hline Imágenes & 6,0 \\
\hline Hospitalización maternidad & 5,4 \\
\hline Endoscopía & 4,0 \\
\hline Pediatría & 3,3 \\
\hline Salud mental & 3,2 \\
UPCA * & 2,7 \\
\hline Otros ** & 9,6 \\
\hline
\end{tabular}

*Unidad de Paciente Crítico Adulto. ${ }^{* *}$ Incluye: Laboratorio Clínico, Neonatología, Odontología, Unidad de Terapia Física.

se llevaron a efecto una o más entrevistas presenciales con el paciente, un familiar o el responsable legal de éste. El 3,5\% de los reclamos derivó a una mediación luego de una presentación ante la Superintendencia de Salud, y 1\% de los reclamos no logró resolución en alguna etapa previa y motivó el inicio de una demanda contra el profesional y/o la institución. El 97\% de los reclamos se resolvió dentro de los primeros cinco días hábiles posteriores a su recepción.

\section{Discusión}

La información recogida en este trabajo enfrenta varias situaciones especiales. La primera de ellas es que no son bien conocidas, en nuestro medio, las tasas y causas de los reclamos médicos. Habitualmente, los reclamos son tratados al interior de las instituciones prestadoras de salud y no son divulgados. En nuestra experiencia, se ha generado un reclamo por cada 1.000 prestaciones, cualquiera sea el tipo, de forma bastante estable durante el período analizado. No conocemos de otras fuentes de información para poder comparar esta observación con lo que ocurre en otros centros privados.

Publicaciones recientes han presentado los
Tabla 4. Causa de los reclamos

\begin{tabular}{|lcc|}
\hline Causa & n & \% \\
Falla en relación con médico & 129 & 21,4 \\
Cobros/presupuestos/coberturas & 126 & 20,9 \\
Expectativas & 75 & 12,5 \\
Falla en relación con enfermería u & 60 & 10,0 \\
otros profesionales & 57 & 9,5 \\
Falla/retardo en el diagnóstico & 37 & 6,1 \\
Falla/retardo en el tratamiento & 34 & 5,7 \\
Descoordinación & 31 & 5,1 \\
Evento adverso & 26 & 4,3 \\
Complicación de tratamiento médico/ & & \\
quirúrgico/parto & 25 & 4,2 \\
\hline Otros & 2 & 0,3 \\
\hline Error asociado a medicamento & & \\
\hline
\end{tabular}

Tabla 5. Forma de resolución del reclamo

\begin{tabular}{|lcc|}
\hline Resolución del reclamo & n reclamos & \% \\
\hline A: llamada telefónica & 450 & 74,7 \\
\hline B: entrevista & 125 & 20,8 \\
C: mediación & 21 & 3,5 \\
\hline D: juicio & 6 & 1,0 \\
\hline
\end{tabular}

incidentes que produjeron un reclamo ante el Consejo de Defensa del Estado ${ }^{7}$, en el ámbito de las prestaciones a nivel público. De acuerdo a esa información se presume que inicialmente fueron reclamos al interior de las instituciones, las que derivaron en una mediación, atendida finalmente por este último organismo. Sobre el análisis de 10.409 reclamos, en la publicación de Lagos et $\mathrm{al}^{7}$, se señala que $23,6 \%$ fue motivado por un retardo en la atención, otro $23 \%$ por un error diagnóstico y $19 \%$ por lo catalogado como "un error en la realización de procedimientos quirúrgicos o de enfermería”. Es aquí donde nos encontramos con otra de las dificultades en el análisis de esta realidad. No existe un listado universal y sistematizado de los incidentes que pueden llevar a un reclamo, que pueden ser muy variados. Ello no quiere decir que 
no puedan ser clasificables en algunas principales categorías, que sean adoptadas por otros centros y así facilitar las comparaciones. La Superintendencia de Salud, el año $2018^{1}$ señaló que $36 \%$ del total de reclamos, sumando sector público y privado, se debió a la falta de una atención oportuna y sin discriminación. El 28\% fue por falta de información administrativa y/o financiera; $14 \%$ por falla en obtener un trato digno y en $11 \%$ por la falta de información clínica, por mencionar las causales más relevantes.

Al tomar la información de la Superintendencia de Salud ${ }^{1}$ sobre las materias reclamadas en el ámbito privado, las causas que involucran directamente al médico son aquellas de falla en trato digno y en dar información clínica y ambas suman $24 \%$. La falla en dar atención oportuna y sin discriminación, puede comprometer a otros profesionales de la salud y no se han sumado para este efecto. Para el sector público, los reclamos que involucran al médico llegan $31 \%$. En nuestro análisis $21,4 \%$ de los reclamos, se debió a una falla en la relación con el médico (comunicación, trato, empatía, amabilidad), que guarda relación con la experiencia del Hospital Clínico de la Pontificia Universidad Católica ${ }^{6}$, donde se señala que 19\% de 8.931 reclamos involucró a un médico. De esta última experiencia, $51 \%$ reconoció como causa un conflicto en la relación médico-paciente, siendo la comunicación un punto crucial. Parece, por tanto, de la mayor importancia, poner atención y corregir todos aquellos quiebres en tal relación, sobre la que se fundamenta el acto médico. Tal como ha sido señalado ${ }^{3,8}$ y dado que una comunicación adecuada es susceptible de ser entrenada y está en base de esa buena relación, parece fundamental reforzar esa habilidad con formación específica en el pregrado y posteriormente. Como lo señalan los autores del referido artículo ${ }^{6}$ ellos han implementado cursos formales sobre tales materias, lo que también ha desarrollado nuestra Escuela de Medicina.

Reclamos relacionados específicamente a los cobros, presupuestos y coberturas, nuestra segunda causa por frecuencia, con $20,9 \%$ se compara favorablemente a lo señalado por la Superintendencia de Salud ${ }^{1}$, cuando realiza el análisis general del sistema privado, en que señala $48 \%$. Para el sistema público, la falta de información administrativa y/o financiera es causa de reclamo sólo en $5 \%$ de los casos ${ }^{1}$.
En nuestra experiencia, han sido infrecuentes causas de reclamo los "errores asociados a medicamentos", las "complicaciones de un tratamiento médico y/o quirúrgico" y las "fallas o retardo en el tratamiento o en el diagnóstico". Como explicación de lo primero, pudiera ser el rol que juega el registro clínico electrónico y la obligatoriedad que imprime un sistema informático para la solicitud, administración y registro de fármacos. La baja frecuencia de presentarse un reclamo frente a una complicación de un tratamiento médico o quirúrgico, puede hablar de una adecuada relación médico-paciente, una vez ocurrida ésta.

En cuanto a la resolución del conflicto, en nuestra serie, $75 \%$ de los reclamos se resolvió mediante una llamada telefónica, y 21\% tras una o más entrevistas con el paciente, un familiar o el responsable legal. Estamos ciertos que, con el contacto personal, se da la instancia de lograr, en un ambiente de mutuo respeto, la comprensión de lo sucedido, la ocasión de ofrecer las disculpas por ello y extender la promesa de corregir los procesos y políticas de atención, para evitar que vuelva a ocurrir lo mismo, como ha sido bien señalado ${ }^{5}$. En ese sentido, la mediación es una estupenda instancia para lograr los objetivos recién expuestos, evitando escalar a la judicialización ${ }^{9,10}$. No obstante, $y$ en un período de más de diez años, la experiencia en Chile es que se llega a un acuerdo tan sólo en $21 \%$ de los casos expuestos a una mediación ${ }^{7}$. Es positivo observar, sin embargo, que, a lo largo de los años, han aumentado los acuerdos luego de detallarse las explicaciones y dar las disculpas correspondientes, sobre acuerdos logrados bajo la exclusiva indemnización económica ${ }^{5}$. En nuestra experiencia sólo 3,5\% de los reclamos no fue resuelto al interior de la institución y se requirió de la participación de un mediador en salud. En 6 casos $(1 \%)$ de los 602 reclamos, se inició una demanda judicial. Se ha señalado que alrededor de $10 \%$ de quienes no llegan a acuerdo a través de un proceso de mediación, posteriormente inician acciones judiciales indemnizatorias ${ }^{5,11}$.

Nos parece relevante conocer con detalle las materias que llevan a presentar un reclamo asociado a la atención en salud, como una forma efectiva de poder corregirlas. Más aún, cuando parecieran ser diferentes si el incidente se ha producido en el sistema público de salud o en el entorno de un prestador privado de salud. Urge llegar a un consenso sobre cómo clasificar las causas, de modo de 
poder establecer estudios comparativos y poder hacer un seguimiento y conocer el impacto de los planes de mejora, tendientes a corregir las causas, en especial aquellas más frecuentes.

Finalmente, dado que, en el contexto social actual, el respeto por el otro se sitúa como uno de los principales valores a defender y procurar, existe un gran espacio para reducir las tasas de reclamos debido a lo que hemos llamado falla en el trato y comunicación con el médico y que en otras publicaciones son englobadas como menoscabo en el "trato digno"6, 12-14. Debemos reforzar la formación integral de los médicos, con el fin de que las situaciones de conflicto por asuntos relativos a las relaciones humanas, que se dan en el binomio paciente-médico, se minimicen y eventualmente, desaparezcan ${ }^{15}$.

\section{Referencias}

1. Gestión de Reclamos como una Herramienta para la Mejora Continua. Subdepartamento de Derechos de las Personas. Intendencia de Prestadores. Superintendencia de Salud. Chile 2018. https://www.supersalud.gob.cl/observatorio/671/articles-17372_recurso_6.pdf

2. Ley $n^{\circ} 20.584$ de Derechos y Deberes, que "regula los derechos y deberes de las personas en relación con acciones vinculadas a su atención de salud". Diario Oficial, 24 de abril, 2012. http://www.supersalud.gob.cl/normativa/668/ articles-7564_recurso_1.pdf

3. Osorio G, Sayes N, Fernández L, Araya E, Poblete D. Audi- toría médica: herramienta de gestión moderna subvalorada. Rev Med Chile 2002; 130: 226-9.

4. Ley $n^{\circ}$ 19.966. Régimen General de Garantías en Salud. Diario Oficial, 3 de septiembre, 2004. https://www.supersalud. gob.cl/normativa/668/w3-article-554.html

5. Munuera P. La mediación sanitaria en Chile. Rev Med Chile 2020; 148: 792-8.

6. Moore P, Vargas A, Núñez S, Macchiavelo S. Un estudio de reclamos hospitalarios: el rol de la relación médico-paciente. Rev Med Chile 2011; 139: 880-5.

7. Lagos D, Bravo L. Mediación en el sistema público de salud: oportunidades para mejorar. Rev Med Chile 2020; 148: 2115.

8. Rodríguez Almada H. De la medicina defensiva a la medicina asertiva. Rev. Méd. Urug. [Internet]. 30 de septiembre de 2006 [citado 5 de mayo de 2021]; 22 (3): 167-8.

9. Armadans I, Aneas S, Soria MA, Bosh L. La mediación en el ámbito de la salud. Med Clin. (Barc) 2009; 133: 187-92.

10. Wilkinson D, Barclay S, Savulescu J. Disagreement, mediation, arbitration: resolving disputes about medical treatment. Lancet. 2018 Jun 9; 391 (10137): 2302-5. doi: 10.1016/S0140-6736 (18) 31220-0

11. Lagos MS. Impacto de la mediación en la judicialización de conflictos jurídicamente relevantes: un análisis en base a la experiencia chilena de mediación por daños de salud. Revista de Estudios de la Justicia Chile 2013; 18: 83-110.

12. Ceriani JM. Medical practice in the technological age. Arch Argent Pediatr. 2017; 115: 106-7.

13. Olivares P. Empatía y cuidado profesional: esbozos de una relación problemática. Ars Medica 2020: 45: 55-61.

14. Espinoza R. El paciente difícil y profesionalismo médico. Rev Cir. 2019; 71: 270-3.

15. Navarrete G. ¿Es suficiente la base moral de los médicos? Rev Med Chile 2020; 148: 684-8. 